\title{
Methodology for GPS Synchronization Evaluation with High Accuracy
}

\author{
Zan Li, Torsten Braun, Desislava C. Dimitrova \\ Institute of Computer Science and Applied Mathematics, University of Bern, Bern - Switzerland \\ Email:1i@iam.unibe.ch, braun@iam.unibe.ch, dimitrova@iam.unibe.ch
}

\begin{abstract}
Clock synchronization in the order of nanoseconds is one of the critical factors for time-based localization. Currently used time synchronization methods are developed for the more relaxed needs of network operation. Their usability for positioning should be carefully evaluated. In this paper, we are particularly interested in GPS-based time synchronization. To judge its usability for localization we need a method that can evaluate the achieved time synchronization with nanosecond accuracy. Our method to evaluate the synchronization accuracy is inspired by signal processing algorithms and relies on finegrain time information. The method is able to calculate the clock offset and skew between devices with nanosecond accuracy in real time. It was implemented using software defined radio technology. We demonstrate that GPS-based synchronization suffers from remaining clock offset in the range of a few hundred of nanoseconds but the clock skew is negligible. Finally, we determine a corresponding lower bound on the expected positioning error.
\end{abstract}

\section{INTRODUCTION}

Localization and tracking have emerged in recent years as an attractive solution to enable new business models that rely on personalized provisioning of location-based services. Positioning approaches relying on radio technologies, such as WiFi and Global System of Mobile communication (GSM), have been proposed for localization in dense urban areas and indoor spaces [1], where the usability of the Global Positioning System (GPS) is limited [2]. Time Difference of Arrival (TDOA) is one of the promising source localization algorithms based on arrival time differences of the signal from an object at different Anchor Nodes (ANs) [2], [3]. In reality the process is challenged by imperfect time synchronization due to the high propagation speed of the signal. Since perfect synchronization is unrealistic in practice, we need to (1) identify appropriate synchronization solutions and (2) develop methods to quantify the achieved degree of synchronization. Our choice of GPSbased synchronization is driven by its theoretically expected accuracy in the order of nanoseconds [4]. Unfortunately, current methods to evaluate the time synchronization either suffer from low accuracy in the range of microseconds or depend on expensive devices, e.g., a digital analyser.

In this paper, we first describe a method based on the timing error in the signal processing procedure to calculate the remaining clock offsets with accuracy in the range of nanoseconds. To extract the timing error information, we adopt software defined radio technology, which provides a flexible way to work on signal processing. Second, in practice, measurements of clock offsets are noisy and, thus, it is challenging to calculate its differentiation, i.e., clock skew. We propose to adopt Savitzky-Golay (S-G) filter [5] to smooth the noisy measured clock offset and then accurately calculate the clock skew. Finally, based on the timing error information, we investigate the duration of the initialization phase for GPS synchronization. Compared to traditional methods, we are able to: (1) measure the clock offset and skew between two GPS synchronized devices with nanosecond accuracy, (2) calculate the clock offset and skew in real time, and (3) not depend on any additional measurement device, e.g., digital analyser. All of these are critical requirements in a time-based localization system.

Through a set of comprehensive measurements, we find that GPS as the most promising synchronization approach has shortcomings and sets a limit on positioning accuracy to tens of meters. However, clock skew between the two GPS synchronized devices is negligible. Our findings are relevant for all TDOA-based localization schemes independent of the used radio signal and other applications with high requirements on accurate synchronization, e.g., time synchronization in Long-Term Evolution Time-Division Duplex (LTE TDD) and later LTE-Advanced networks.

Our study is conducted in the scope of TDOA-based source localization with GSM signals. Along with synchronization, the calculation of a highly accurate timestamp by the ANs is critical for TDOA localization. The process is particularly challenging with GSM (narrow-band) signals, given the strong impact of multipath propagation. An independent, exhaustive study on the topic is addressed in our current work, and we show preliminary insights on the influence of multipath propagation on the synchronization evaluation in this paper.

In the following sections, we first introduce GPS synchronization in Section II-A, followed by an introduction to clocks and quantitative approaches to evaluate clock synchronization (Sections II-B and II-C). The proposed methods to analyze the synchronization accuracy are presented in Section III. Sections IV and V cover its implementation and evaluation, respectively. Finally, Section VI concludes the paper.

\section{SYNCHRONIZATION IN LOCALIZATION SYSTEMS}

\section{A. GPS Synchronization}

In a large testing area where the ANs can not be synchronized by a shared cable, GPS offers the most promising synchronization solution for localization due to the accurate system time regularly provided by satellites. GPS receivers attached to nodes on the ground can synchronize their local clocks to signals from GPS satellites. There is a Phase Lock Loop (PLL) with an inaccurate local clock inside each GPS receiver. PLL requires a certain time to lock to the GPS signals, denoted as an initialization phase. After PLL has locked, PLL 
TABLE I: Notation list

\begin{tabular}{|c|c|c|c|}
\hline$\Delta C_{i j}$ & Relative clock offset & $\mu$ & Normalized timing error \\
\hline$\Delta f_{i j}$ & Relative clock skew & $\bar{\mu}$ & Averaged normalized timing error \\
\hline$\Delta \theta_{i j}$ & Relative initial clock offset & $\Delta \mu_{i j}$ & Relative normalized timing error \\
\hline
\end{tabular}

steps into a stable phase. In the stable phase, PLL needs to be periodically adjusted based on the received GPS signals. If the GPS receivers can not receive the signals from the satellites, the local clocks will continuously drift away from each other. Hence, indoor devices should be synchronized by using GPS receivers with outdoor antennas.

\section{B. Clock Terminology}

Every AN in a time-based localization system has its own clock. Assuming that the reference time of the whole system is $C(t)=t$, ideally, the local time of the $i$ th $\mathrm{AN}\left(A N_{i}\right)$ should be $C_{i}(t)=t$. However, even if started at exactly the same time, local clocks will drift away from the reference time because of frequency deviations of the oscillator.

Generally, the clock function of $A N_{i}$ is modeled as

$$
C_{i}(t)=\int_{0}^{t} \Delta f_{i}(t) \mathrm{d} t+\Delta \theta_{i}
$$

where the parameters $\Delta f_{i}(t)$ and $\Delta \theta_{i}$ are the clock skew and initial clock offset at $A N_{i}$. In this paper we use the nomenclature from [6] to define that $\Delta C_{i j}(t)=C_{i}(t)-C_{j}(t)$ is the relative clock offset, and $\Delta f_{i j}(t)=\Delta f_{i}(t)-\Delta f_{j}(t)$ is the relative clock skew between $A N_{i}$ and $A N_{j}$. Table I introduces the notations used in the paper.

\section{Quantifying Synchronization Accuracy}

One method to determine whether two devices (e.g., ANs) are synchronized is to compare their timestamps for the same receiving message emitted by an equidistant reference node. In a real wireless network the timestamp for the received message reflects the signal propagation time as well as the time spent in receiving and processing the message at the receiver node [7], [8]. Hence, a timestamp is best given close to the physical layer to avoid influences from processing time at the Media Access Control (MAC) or higher layers. At the physical layer, a conventional timestamp [9] is the hardwaregiven time when a sample of a packet is received, e.g., the last or first sample of a training sequence. The conventional timestamp is limited by signal properties such as bandwidth and symbol rate. In the case of a GSM signal with symbol rate of $270.8 \mathrm{KHz}$, a conventional timestamp can not distinguish time differences within one symbol interval $(3.7 \mu \mathrm{s})$ and thus the corresponding timestamp resolution is only $3.7 \mu \mathrm{s}$.

\section{Synchronization Accuracy VIA Time Recovery}

The quality of the sampling process directly affects the quality of the taken timestamp. Several methods have been developed to improve the sampling process for signal recovery, such as time recovery [10]. We go one step further and exploit the method of the time recovery to achieve highly accurate calculation of the synchronization misalignment among nodes.

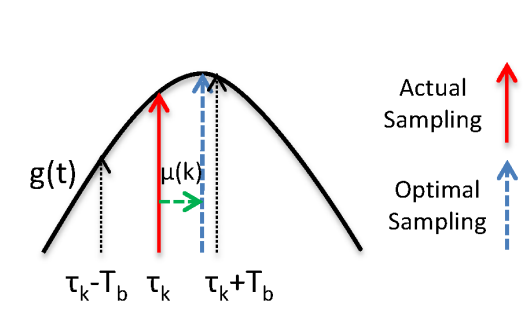

(a) Adjusting Sampling Position

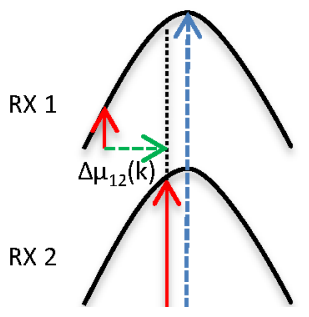

(b) Time Offset
Fig. 1: Time Recovery

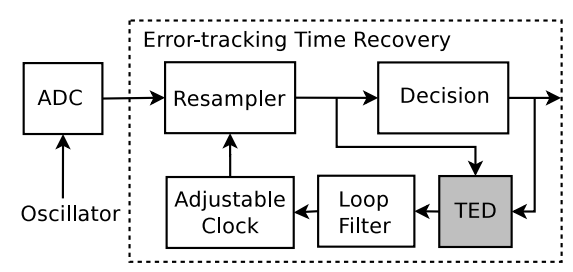

Fig. 2: Digital Architecture of Time Recovery Loop

\section{A. Pulse Sampling and Time Recovery}

In an ideal system, where the transmitter and receiver are perfectly synchronized, timestamps are taken at the optimal sampling moment of a symbol. In the case of a GMSK modulation system, as applied in GSM, the signal is shaped by a Gaussian filter and thus the optimal sampling position is at the peak of the Gaussian pulse $g(t)$ as shown in Figure 1(a). Denoting the sampling phase by $\tau_{k}$, sampling would be on the peak of the pulse when $g\left(\tau_{k}-T_{b}\right)=g\left(\tau_{k}+T_{b}\right)$, where $T_{b}$ is the symbol interval [10]. The reason for this is the evenly symmetric shape of the Gaussian pulse.

In practical systems, however, the receiver is not synchronous with the incoming data due to free running oscillators of the Digital-to-Analogue Converter (DAC) at the transmitter and the Analogue-to-Digital Converter (ADC) at the receiver. This results in suboptimal sampling, i.e., the actual sampling position is often before (as in Figure1(a)) or after the peak, displaced at $\mu(k), \mu(k)$ is the normalized timing error given by:

$$
\mu(k)=\frac{\Delta T(k)}{T_{s}},
$$

where $\Delta T(k)$ is the offset between the actual and optimal sampling positions and $T_{s}$ is the constant sampling interval.

If two co-located receivers are not perfectly synchronized with each other, the shift in sampling position, $\mu(k)$, will be different for each receiver. We denote $\Delta \mu_{12}(k)$ as the difference in $\mu(k)$ between two receivers as shown in Figure 1(b). The better we can calculate $\Delta \mu_{12}(k)$, the more accurately we can evaluate the degree of synchronization. 
Figure 2 shows the architecture of an error tracking time recovery loop, which is adopted to correct the shift in the sampling position [10]. The sample stream is fed into a Timing Error Detection (TED) module to extract the timing error information between the actual and optimal sample positions. The timing error information is passed to a loop filter, which outputs the normalized timing error $\mu(k)$ to decide on the correction of the sampling time in the re-sampler. Subsequently, the sampling position can be adjusted to be closer to the optimal one.

\section{B. Relative Clock Offset and Skew Calculation}

In this subsection, we will derive the relation between the normalized timing error $\mu(k)$, the clock offset and skew. At the physical layer a conventional timestamp $T^{\prime}(k)$ for the $k t h$ sample from the beginning of the sample stream can be obtained as follows [9]:

$$
T^{\prime}(k)=T^{\prime}(1)+T_{s} *(k-1),
$$

where $T^{\prime}(1)$ is the conventional timestamp for the first sample in the stream. Equation (3) indicates that the conventional timestamp can only change at a step of $T_{s}$ and hence the resolution is limited by $T_{s}$. With $\mu(k)$ obtained by the time recovery, we can improve the resolution as:

$$
T(k)=T^{\prime}(k)+\mu(k) \cdot T_{s},
$$

where $T(k)$ is the improved timestamp. Assuming that the first sample of the received packet is the kth sample from the beginning of the sample stream, $T(k)$ is the improved timestamp for this packet.

1) Relative Clock Offset without Long Term Clock Drift: GPS synchronization is well known to not have long-term clock drift. In the case that the sampling interval $T_{s}$ is in the range of microseconds and GPS synchronization offset is smaller than one microsecond, the clock offset between two ANs is smaller than the sampling interval $T_{s}$ at any moment. In such case, we can derive the relation between the relative clock offset and the relative normalized timing error. Based on Equation (4) and the definition of relative clock offset, we can obtain the relative clock offset between the $i$ th and $j$ th receivers,

$$
\begin{aligned}
\Delta C_{i j}(k) & =T_{i}(k)-T_{j}(k) \\
& =\left(T_{i}^{\prime}(k)+\mu_{i}(k) \cdot T_{s}\right)-\left(T_{j}^{\prime}(k)+\mu_{j}(k) \cdot T_{s}\right) .
\end{aligned}
$$

If the relative clock offset is always smaller than the sampling interval $T_{s}$, we can get that $T_{i}^{\prime}(k)=T_{j}^{\prime}(k)$ for the same packet $k$. Therefore, we can further simplify Equation (5) as,

$$
\begin{aligned}
\Delta C_{i j}(k) & =\mu_{i}(k) \cdot T_{s}-\mu_{j}(k) \cdot T_{s} \\
& =\Delta \mu_{i j}(k) \cdot T_{s},
\end{aligned}
$$

where $\Delta \mu_{i j}(k)=\mu_{i}(k)-\mu_{j}(k)$. In the above equation, $T_{s}$ is constant and, thus, the relative clock offset is determined by $\Delta \mu_{i j}(k)$.
Furthermore, considering Equation (1) and $t=k \cdot T_{s}$, we can calculate

$$
\begin{aligned}
\Delta C_{i j}(t) & =T_{s} \cdot \Delta \mu_{i j}(t) \\
& =\int_{0}^{t} \Delta f_{i j}(t) \mathrm{d} t+\Delta \theta_{i j},
\end{aligned}
$$

where $\Delta \theta_{i j}$, the relative initial clock offset between $A N_{i}$ and $A N_{j}$, is constant. Therefore, we can analyze the shortterm clock drift through the relative normalized timing error $\Delta \mu_{i j}(t)$. If there is no short-term clock drift, $\Delta \mu_{i j}(t)$ would be constant. Otherwise, $\Delta \mu_{i j}(t)$ would fluctuate. We can use this to identify the presence of short-term clock drift by monitoring the behavior of $\Delta \mu_{i j}(t)$.

2) Relative Clock Skew via Savitzky-Golay Filter: Here we derive how to obtain the relative clock skew between two ANs. Based on Equation (7), we can obtain the relative clock skew in an ideal case as

$$
\Delta f_{i j}(t)=\frac{\mathrm{d}}{\mathrm{d} t}\left(\Delta C_{i j}(t)\right)=T_{s} \cdot \frac{\mathrm{d}}{\mathrm{d} t}\left(\Delta \mu_{i j}(t)\right) .
$$

Therefore, the relative clock skew can be calculated by the differentiation of $\Delta C_{i j}(t)$ or $\Delta \mu_{i j}(t)$.

However, in practice, the measurement of $\Delta \mu_{i j}(t)$ is noisy and it is hard to accurately measure its differentiation. Therefore, in our work, we propose to estimate the relative clock skew in two steps.

At the first step, an S-G filter [11] is applied to smooth the measured $\Delta \mu_{i j}(t)$. The $\mathrm{S}-\mathrm{G}$ filter applies a moving window smoothing technique based on least squares polynomial fitting. We take the group of $2 M+1$ samples of $\Delta \mu_{i j}(t)$ centered at $n$, which is moving from 0 to the end of the samples. We obtain the coefficients of a polynomial,

$$
\Delta \mu_{i j}^{\prime}(n)=\sum_{j=0}^{N} a_{j} n^{j},
$$

which minimizes the mean-squared approximation error,

$$
\begin{aligned}
\varepsilon_{N} & =\sum_{n=-M}^{M}\left(\Delta \mu_{i j}^{\prime}(n)-\Delta \mu_{i j}(n)\right)^{2} \\
& =\sum_{n=-M}^{M}\left(\sum_{j=0}^{N} a_{j} n^{j}-\Delta \mu_{i j}(n)\right)^{2},
\end{aligned}
$$

where $N$ is the order of polynomial and $2 M+1$ is the size of the moving window. The coefficients $\left[a_{0}, a_{1}, \ldots, a_{N}\right]$ are adaptive in each window. $\Delta \mu_{i j}^{\prime}(n)$ is the output of the filter and sampled $\Delta \mu_{i j}^{\prime}(t)$ with sampling space of $T_{s}$.

At the second step, the differentiation of the filtered value $\Delta \mu_{i j}^{\prime}(t)$ or $\Delta C_{i j}^{\prime}(t)$ will be calculated to obtain the relative clock skew as follows,

$$
\Delta f_{i j}(t)=\frac{\mathrm{d}}{\mathrm{d} t}\left(\Delta C_{i j}^{\prime}(t)\right)=T_{s} \cdot \frac{\mathrm{d}}{\mathrm{d} t}\left(\Delta \mu_{i j}^{\prime}(t)\right) .
$$

\section{TIMING INFORMATION EXTRACTION IN SDR}

\section{A. Hardware}

In our evaluations, we use Software Defined Radio (SDR) implementations for the transmitter (packet source) and receivers (ANs). We use the Universal Software Radio Peripheral 


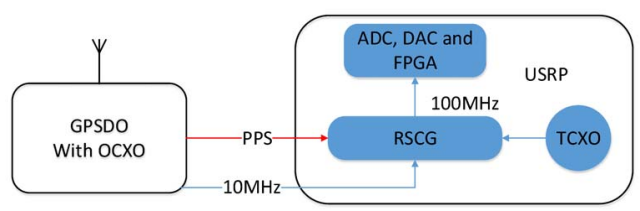

(a) GPS Synchronization Structure

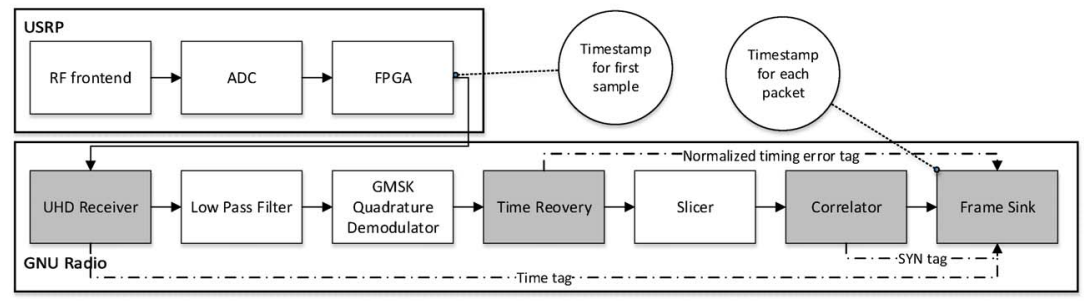

(b) GMSK Receiver with Enhanced Timestamp

Fig. 3: Hardware and Software Realization

(USRP) hardwares [12] by Ettus Research and the open-source GNU Radio software set. USRP comprises of a daughterboard and a motherboard. The daughterboard is a RF frontend to receive and transmit analogue signals. The motherboard is for signal processing, including ADC, DAC, and a Field Programmable Gate Array (FPGA). For our measurements, we use the networked USRP N210 model, which can connect to a desktop by Gigabit Ethernet.

The operation of USRP is controlled by a Reference and System Clock Generation module (RSCG) as shown in Figure 3(a). Ettus Research provides a GPS Disciplined Oscillator (GPSDO) kit [13] with an Oven-Controlled Crystal Oscillator (OCXO), allowing the device to lock to GPS signals.

\section{B. Software Realization}

Figure 3(b) illustrates the components of the SDR system in detail. We acquire the timestamp for the first sample in the sample stream from the FPGA. As mentioned in Section III-B1, the conventional timestamp is limited by the sampling rate. Utilizing the information provided by the time recovery block is proposed to calculate the improved timestamp. For this reason, we need to ensure that the normalized timing error $\mu(t)$ is associated to each sample to calculate the improved timestamp. Therefore, certain modifications of the SDR structure are needed. The modified blocks are indicated in grey in Figure 3(b). We make use of a mechanism called stream tags, provided in GNU Radio to attach tags with control information to samples of the data stream.

First, a time tag, which indicates the starting time of the stream, is attached to the first sample of the stream in the USRP Hardware Driver (UHD) receiver. All following samples are numbered relative to the first sample. Second, we modify the time recovery block to allow forwarding the normalized timing error $\mu(k)$ for each output sample. Third, a SYN tag indicating the first sample in the packet is passed to the frame sink. The combination of the three tags is adopted to extract the normalized timing error $\mu(k)$ for each packet and calculate the improved timestamp according to Equation (4).

\section{Measurement Results and Analysis}

\section{A. Measurement Setup}

To investigate the performance of GPS synchronization, the setup in Figure 4(a) is adopted. Assuming the same delays in hardware two factors can cause time offset between two receivers, namely, multipath propagation and synchronization

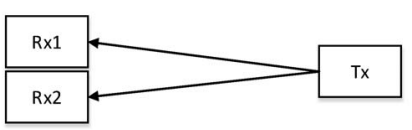

(a) Measurement Setup 1

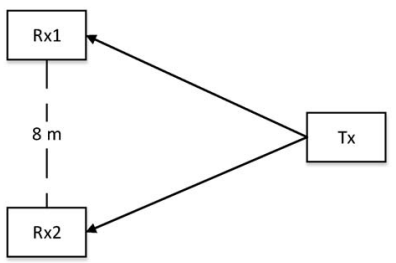

(b) Measurement Setup 2
Fig. 4: Measurement Setup

offset. In order to isolate only the effect of the synchronization component on our measurement, we co-locate the two receivers. This setup ensures as much as possible the same propagation path of the signal. The setup of Figure 4(b) is used to investigate the effects of multipath propagation.

In order to represent the GSM signal, we construct our own transmitter to continuously generate GMSK-modulated signals with $250 \mathrm{KHz}$ symbol rate, which should be an integer divisor of the clock rate in ADC and DAC, $100 \mathrm{MHz}$. The signal is with the same modulation scheme as GSM but operated in the ISM band $(433 \mathrm{MHz})$. At the receiver side, we configure the sampling rate to $500 \mathrm{KHz}$. The corresponding sampling interval $T_{s}$ is $2 \mu \mathrm{s}$. Each packet has a length of 1502 bits. To minimize the noise and ease result presentation, we work with the value $\bar{\mu}$ averaged over the $\mu(k)$ of all the samples in each packet (Table I).

\section{B. GPS Synchronization Evaluation}

Taking the setup of Figure 4(a), we first try to calculate the GPS synchronization offset between the two receivers based on the conventional timestamps (Equation (3)). The measurements do not show a long-term clock drift, at least not larger than the sampling interval $(2 \mu s)$. This observation demonstrates the assumption in Section III-B that clock offsets between two ANs are not larger than the sampling interval $T_{s}$ at any moment. However, due to the limited conventional timestamp resolution, we are not able to determine whether there is a short-term clock drift between the receivers based on the conventional timestamp.

To analyze the performance of GPS synchronization in depth, we adopt the proposed method that uses the normalized timing error $\bar{\mu}$ from time recovery. In particular, we investigate the duration of initialization phase, clock offset, and clock skew. 


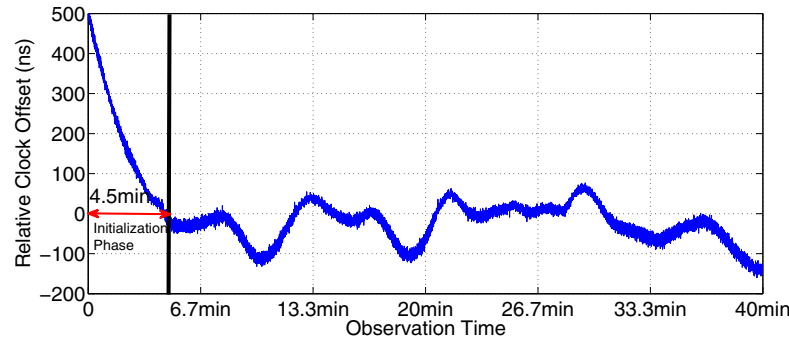

(a) Initialization Phase of GPS Synchronization (M1)

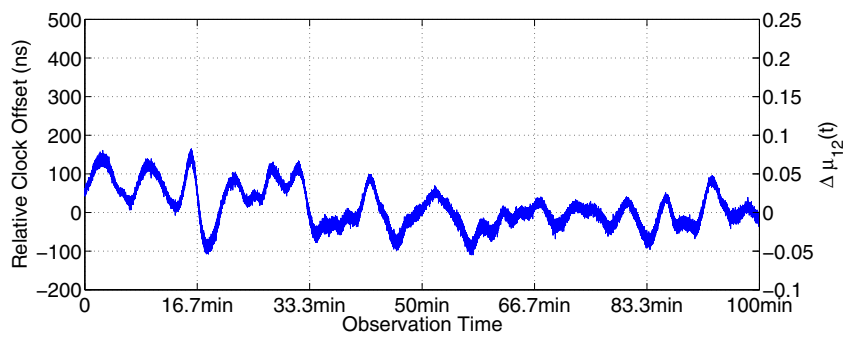

(b) Clock Offset in the Stable Phase (M2)

Fig. 5: Clock offset of GPS synchronization with co-located receivers

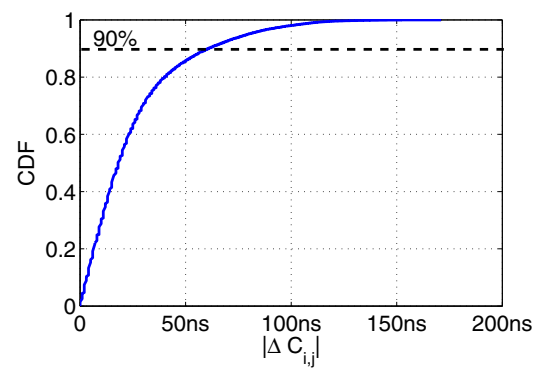

(a) CDF of the Relative Clock Offset (M2)

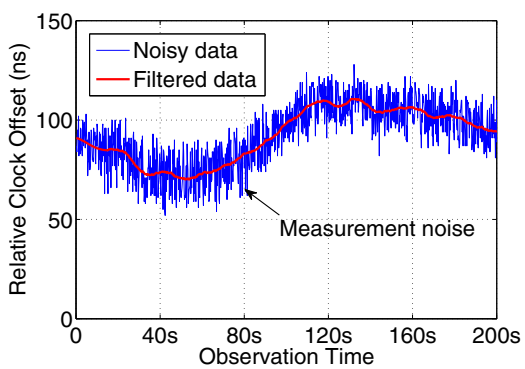

(b) Data Filtering with S-G Filter (M2)

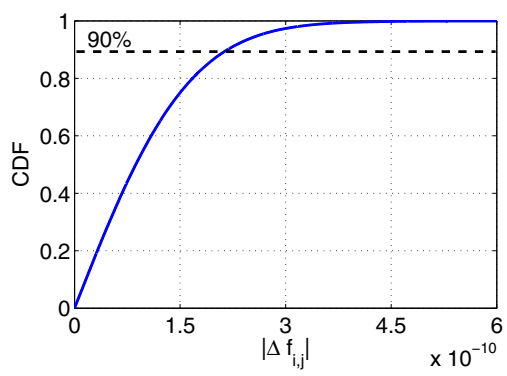

(c) CDF of the Relative Clock Skew (M2)

Fig. 6: Measurement Results for Clock Offset and Skew

Initialization Phase. It is important to measure the length of the initialization phase, during which applications with high requirements of accurate synchronization, e.g., localization, should be avoided. The first measurement denoted as M1 is conducted to measure the length of the initialization phase. With our proposed method, we start to record the clock offset as soon as the two GPS receivers are powered on. Figure 5 (a) indicates the changes of the relative clock offset between two GPS receivers over 40 minutes. We find that the GPS receivers need around 4.5 minutes to synchronize with each other. After the initialization phase, the clock offset between two GPS receivers becomes stable.

Clock Offset. We take a second measurement with duration of 18 hours to analyze the GPS clock offset in the stable phase. The measurement is denoted as M2. In the measurement, the $\Delta \mu_{12}(t)$ values are collected 5 minutes after the devices have been started to avoid the initialization phase in the GPS receivers.

Figure 5(b) indicates parts of the measurement results, where the left Y-axis indicates the relative clock offset and the right $\mathrm{Y}$-axis is the value of $\Delta \mu_{12}(t)$. The relative clock offset between two GPS receivers can be calculated based on Equation (6) where the sampling interval $T_{s}$ is $2 \mu s$. Fluctuations in the $\Delta \mu_{12}(t)$ value corresponding to the variation in the relative clock offset is well visible. Figure 6(a) summarizes the Cumulative Distribution Function (CDF) of the absolute value of the clock offset $\left(\left|\Delta C_{i, j}\right|\right)$. The first row in Table II summarizes the relative clock offset between two GPS receivers in the measurement. Mean and Standard Deviation (SD) are calculated for the real value of $\Delta C_{i, j}$, while maximum and $90 \%$ accuracy are for the absolute value $\left(\left|\Delta C_{i, j}\right|\right)$. Our findings show that the maximum measured clock offset between the two receivers is $171 \mathrm{~ns}$, resulting in more than $51 \mathrm{~m}$ localization error for time-based localization. The $90 \%$ synchronization accuracy is $66 \mathrm{~ns}$, corresponding to $19.8 \mathrm{~m}$ localization error for time-based localization.

TABLE II: Relative Clock offset $\left(\Delta C_{i, j}\right)$ between Two GPS Receivers

\begin{tabular}{|c|c|c|c|c|}
\hline Measurement & Mean & SD & Maximum & $90 \%$ Accuracy \\
& $\Delta C_{i, j}$ & $\Delta C_{i, j}$ & $\left|\Delta C_{i, j}\right|$ & $\left|\Delta C_{i, j}\right|$ \\
\hline M2 & $7 \mathrm{~ns}$ & $37.1 \mathrm{~ns}$ & $171 \mathrm{~ns}$ & $66 \mathrm{~ns}$ \\
\hline M3 & $-69.4 \mathrm{~ns}$ & $65.4 \mathrm{~ns}$ & $228 \mathrm{~ns}$ & $150 \mathrm{~ns}$ \\
\hline
\end{tabular}

Clock Skew. Figure 6(b) shows part of the results from measurement M2. As it can be seen in the figure, measurements introduce noise to the data. As introduced in Section III-B2, noisy data prevent us from accurately calculating the relative clock skew. Therefore, we first apply the S-G filter to smooth the data. We calculate the relative clock skew from the differentiation of the filtered clock offset. Figure 6(c) shows the CDF of the clock skew. $90 \%$ of the measured relative clock skew is smaller than $2.18 \cdot 10^{-10}$ and the maximum is $1.37 \cdot 10^{-9}$. It means that during short intervals the change of the relative clock offset can be ignored. For example, during 10 seconds, $90 \%$ of the relative clock offset are changed within $2.18 \mathrm{~ns}$.

\section{Influence of Multipath Propagation}

To demonstrate that multipath propagation may affect the observed synchronization accuracy, we have taken a third measurement (M3) for 18 hours where the two receivers with 


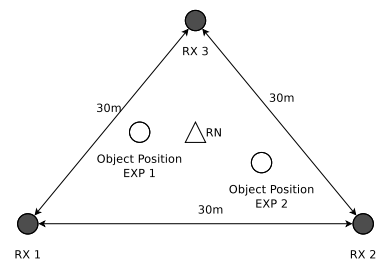

Fig. 7: Measurement Layout

line of sight connection to the transmitter were separated by eight meters in an indoor environment as shown in Figure 4(b). The measurement results are summarized in the second row of Table II. We observe that there is a short-term clock offset between the receivers with deviation of $65.4 \mathrm{~ns}$. More importantly, the mean value of the synchronization offset is $-69.4 \mathrm{~ns}$, which is shifted at a 76.4ns offset compared to the mean value of $7 \mathrm{~ns}$ in the measurement M2. This is caused by multipath propagation.

\section{TDOA Measurement with GPS Synchronization}

We further do measurements to test TDOA accuracy with GPS synchronization. The measurements were conducted in an open space football field to minimize the influence of multipath propagation. The layout of the measurement is given in Figure 7. The improved timestamp of Equation (4) is used to obtain high resolution timestamps of nanoseconds. TDOA values are calculated for two different positions of the object. The Reference Node (RN) is set in the center of the triangle and periodically transmits packets to allow the receivers to check their synchronization offset.

TABLE III: Ranging Error For Open Space Environment

\begin{tabular}{|c|c|c|c|c|}
\hline EXP No. & Algorithms & Rx1 and 2 & Rx2 and 3 & Rx1 and 3 \\
\hline EXP1 & TDOA & $5.6 \mathrm{~m}$ & $22.6 \mathrm{~m}$ & $23.1 \mathrm{~m}$ \\
\hline & SYN offset & $-38 \mathrm{~ns}$ & $-50 \mathrm{~ns}$ & $-77 \mathrm{~ns}$ \\
\hline EXP2 & TDOA & $5.5 \mathrm{~m}$ & $39 \mathrm{~m}$ & $33.5 \mathrm{~m}$ \\
\hline & SYN offset & $-58 \mathrm{~ns}$ & $161 \mathrm{~ns}$ & $105 \mathrm{~ns}$ \\
\hline
\end{tabular}

Table III summarizes the TDOA ranging errors, i.e., the absolute values of the offset between the estimated distance and the real distance. The rows of SYN offset indicate the average synchronization offset between two receivers. For example, for experiment 1 , the synchronization offset of $38 \mathrm{~ns}$ between receiver 1 and 2 means that the clock of receiver 1 is $38 \mathrm{~ns}$ later than receiver 2 . The maximum TDOA ranging error with GPS synchronization reaches $39 \mathrm{~m}$. This further supports our finding that GPS synchronization may generate large synchronization offsets between ANs. Furthermore, based on the packets from the $\mathrm{RN}$, we find that the maximum synchronization offset between the receivers is $161 \mathrm{~ns}$, which is the main reason for TDOA ranging errors in this open space environment. Therefore, calculating and compensating for the remaining GPS synchronization offset is very important for accurate localization.

\section{CONCLUDING REMARKS}

The main contribution of the paper is a method able to evaluate in real-time the degree of GPS synchronization with accuracy in the range of nanoseconds and investigate the performance of GPS synchronization in depth, i.e., initialization phase, clock offset, and clock skew. The main achievements of this paper are (1) an accurate evaluation of the clock offset in case of GPS-based synchronization, and a lower bound of positioning errors set by the maximum synchronization offset of 171ns. (2) The clock skew of GPS synchronization is obtained after filtering the noisy measurement by SavitzkyGolay filter and the maximum clock skew is $1.37 \cdot 10^{-9}$.

\section{REFERENCES}

[1] D. Zhang, F. Xia, Z. Yang, L. Yao, and W. Zhao, "Localization technologies for indoor human tracking," in Future Information Technology (FutureTech), 2010 5th International Conference on, May 2010, pp. $1-6$.

[2] H. Liu, H. Darabi, P. Banerjee, and J. Liu, "Survey of wireless indoor positioning techniques and systems," Systems, Man, and Cybernetics, Part C: Applications and Reviews, IEEE Transactions on, vol. 37, no. 6, pp. 1067-1080, Nov 2007.

[3] Y. Gu, A. Lo, and I. Niemegeers, "A survey of indoor positioning systems for wireless personal networks," Communications Surveys Tutorials, IEEE, vol. 11, no. 1, pp. 13-32, First 2009.

[4] J. yong Yoon, J.-W. Kim, W.-H. Lee, and D.-S. Eom, "A tdoa-based localization using precise time-synchronization," in Advanced Communication Technology (ICACT), 2012 14th International Conference on, Feb 2012, pp. 1266-1271.

[5] R. Schafer, "What is a savitzky-golay filter? [lecture notes]," Signal Processing Magazine, IEEE, vol. 28, no. 4, pp. 111-117, July 2011.

[6] S. Moon, P. Skelly, and D. Towsley, "Estimation and removal of clock skew from network delay measurements,' in INFOCOM '99. Eighteenth Annual Joint Conference of the IEEE Computer and Communications Societies. Proceedings. IEEE, vol. 1, Mar 1999, pp. 227-234 vol.1.

[7] Y.-C. Wu, Q. Chaudhari, and E. Serpedin, "Clock synchronization of wireless sensor networks," Signal Processing Magazine, IEEE, vol. 28, no. 1, pp. 124-138, Jan 2011.

[8] S. Ganeriwal, R. Kumar, and M. B. Srivastava, "Timing-sync protocol for sensor networks," in Proceedings of the 1st International Conference on Embedded Networked Sensor Systems, 2003.

[9] G. Nychis, T. Hottelier, Z. Yang, S. Seshan, and P. Steenkiste, "Enabling mac protocol implementations on software-defined radios," in Proceedings of the 6th USENIX Symposium on Networked Systems Design and Implementation, 2009.

[10] H. Meyr, M. Moeneclaey, and S. Fechtel, Digital Communication Receivers: Synchronization, Channel Estimation, and Signal Processing, 1997.

[11] S. Krishnan and C. Seelamantula, "On the selection of optimum savitzky-golay filters," Signal Processing, IEEE Transactions on, vol. 61, no. 2, pp. 380-391, Jan 2013.

[12] “Usrp n200/n210 networked series data sheet," 2012.

[13] "Gpsdo kit instructions and data sheet," 2012. 